\title{
Technetium-99m-MAG3 renal studies in spinal cord injury patients: normal range, reproducibility, and change as a function of duration and level of injury
}

\author{
WC Klingensmith III, DP Lammertse, DE Briggs, WI Smith, JF Roberts, JW Froelich and JD Sutherland \\ Swedish Medical Center, Craig Rehabilitation Hospital, and Radiology Imaging Associates, Englewood, Colorado, \\ $U S A$
}

The normal range, reproducibility, and change as a function of duration and level of injury for Tc-99m-MAG3 renal studies were quantitated in spinal cord injury (SCI) patients.

METHODS: Five SCI patients without evidence of renal disease in each of four groups: less than 2 months, 2-12 months, 1-2 years, and greater than 2 years from time of injury, were each studied twice. There were at least two patients with paraplegia and two with tetraplegia in each group. Renal clearance (camera based method), percent function in each kidney, time of peak renal parenchymal activity, and half time of parenchmal activity following the peak were evaluated. The peak and half times were determined with regions of interest (ROIs) over the entire kidney and over just the cortex. All results were compared to normal ranges previously established in normal subjects of the same age range using the same methodology. RESULTS: Renal clearance in the less than 2 month SCI patients was not significantly different from normal subjects in either paraplegic or in tetraplegic individuals. However, clearance in tetraplegics was increased by $28.5 \%$ at $2-12$ month, increased by $50.6 \%$ at $1-2$ years, and decreased by $25.9 \%$ at greater than 2 years compared to normal subjects (all $P<0.02$ ). Clearance in those with paraplegia showed a similar, but less marked, trend $(P=\mathrm{NS})$. The time of peak parenchymal activity when measured with cortical ROIs did not vary among patient groups or level of injury, but was increased compared to normal subjects $(P<0.05)$. The percent function in each kidney and half time following the peak were symmetrical, did not differ among patient groups or with level of injury, and did not differ from normal subjects. The parenchymal peak time was significantly shorter with cortical rather than renal ROIs in all patient groups $(P<0.05)$.

In serial studies in the same patient the percent standard deviation in total renal clearance was less than between single studies in different patients, but the decrease was significant for only the right kidney $(P<0.03)$, and the decrease was not as great as in normal subjects. In addition, the percent standard deviation for percent function in each kidney was significantly less than the percent standard deviations in single studies $(P<0.02)$. There were no significant differences between intra- and interpatient studies for any other parameter.

CONCLUSION: We conclude that: (1) renal clearance measured with Tc-99m-MAG3 in tetraplegic patients increases significantly during the first 2 years following injury and decreases significantly thereafter; there is a similar, but much less marked, trend in paraplegics, (2) parenchymal peak times with cortical ROIs occur later for SCI patients than for normal subjects, and (3) there is more intrapatient variation in total renal clearance and percent renal clearance on a side in SCI patients than in normal subjects suggesting that it may be harder to study SCI patients reproducibly. These findings should be taken into account when performing and interpreting Tc-99m-MAG3 renal studies in SCI patients.

Keywords: renal studies; Tc-99m-mercaptoacetyltriglycine (MAG3); tetraplegia; paraplegia; renal clearance; reproducibility

\section{Introduction}

Historically renal disease secondary to complications related to the presence of a neuropathic bladder has been the major cause of death in spinal cord injury (SCI)

Correspondence: WC Klingensmith III, 4720 East Oxford Avenue, Englewood, CO 80110, USA patients. ${ }^{1}$ Although recent advances in bladder management such as intermittent catheterization and bladder retraining have reduced the incidence of renal disease, renal disease remains a frequent complication. ${ }^{1}$ Because of the frequency of renal complications, routine annual evaluations of renal function are commonly performed. ${ }^{2}$ 
Renal scintigraphy with radiopharmaceuticals rather than excretory urography has become the method of choice for evaluating renal function and anatomy in SCI patients because there is no risk of contrast reaction, bowel preparation is unnecessary, and quantitative functional information is available as well as high contrast anatomical information. ${ }^{3}$

The current radiopharmaceutical of choice for renal scintigraphy is Tc-99m-MAG3 because it combines the advantages of a technetium- $99 \mathrm{~m}$ label with the favorable biological properties of a compound that is cleared by tubular secretion. ${ }^{4}$ Renal scintigraphy allows the quantitation of a number of physiologic parameters including renal clearance which traditionally has been reported in terms of effective renal plasma flow (ERPF). Renal clearance may be measured by several different approaches including ones based on blood and urine sampling, blood sampling alone, or simply counts measured in the scintigraphic images (camera based method). We use the camera based method because of its practicality and the likelihood that it will be the most widely utilized approach.

In order to determine the effect of SCI on renal clearance and other physiologic parameters as a function of time from injury we studied four groups of patients with varying durations and levels of SCI who had no evidence of renal disease. In addition, each patient was studied twice to evaluate the reproducibility of renal scintigraphy with Tc-99mMAG3 in SCI patients. All of the results were then compared to normal ranges determined previously in normal subjects of the same age range using the same methodology. ${ }^{5}$

\section{Methods}

\section{Patients}

The study protocol was approved by the institutional ethics committee and informed consent was obtained from all patients. Patients were required to have no history of renal disease and a normal serum creatinine. The urinalysis was allowed to have up to 15 white blood cells per high power field in patients who were not catheterized. Patients were also subsequently excluded if the Tc-99m-MAG3 renal study demonstrated any abnormalities.

Five patients were selected for each of four groups: (1) less than 2 months from the time of SCI, (2) 2-12 months from the time of SCI, (3) 1-2 years from the time of SCI, and (4) greater than 2 years from the time of SCI. Each of the 20 patients was paid to undergo a second Tc-99m-MAG3 renal study in addition to the routine evaluation study. The two studies in each patient were performed at least two days apart.

Patients were not selected for paraplegia or tetraplegia, but at the end of the data collection, when it was realized that each group was nearly evenly divided by level of injury, this variable was also analyzed.
Radiopharmaceutical and imaging system

Each study was performed with approximately $5 \mathrm{mCi}$ of Tc-99m-MAG3 (Mallinckrodt Medical, St. Louis) as previously described. ${ }^{5}$ Images were acquired with either a Picker or Siemens 15 inch field of view gamma camera fitted with a high resolution collimator. The data were analyzed with a Picker 512 Plus or Hitachi Vision computer. The count rate response of each camera/computer system was determined over the salient count rates and normalized to the Picker camera/computer system which was used in the majority of patients.

\section{Imaging method}

The patient was hydrated with at least $600 \mathrm{ml}$ of water one hour prior to the study. ${ }^{6}$ Initially, a one minute image of the syringe containing the dose was obtained with the syringe placed $30 \mathrm{~cm}$ in front of the center of the gamma camera. ${ }^{7-9}$ The patient was then positioned supine over the gamma camera with the kidneys and bladder within the field of view. The dose was injected intravenously using the tourniquet technique. ${ }^{10}$ Serial three second digital images were obtained for the first minute followed by serial one minute digital images for the next 22 minutes; all images were acquired in a 128 $\times 128$ matrix. The acquisition sequence and subsequent analysis were largely determined by software available on the Picker 512 Plus computer.

At the end of the acquisition of the renal images, three additional images were acquired for calculation of the injected dose and for estimation of the percent of injected dose that was infiltrated. One minute images were acquired of the injection site at zero $\mathrm{cm}$ and of the post injection syringe at zero and $30 \mathrm{~cm}$. The ratio of the counts from the syringe at zero and 30 $\mathrm{cm}$, usually 0.95 , was used to normalize the counts from the injection site to the counts of the preinjection syringe. Studies with estimated percent extravasation above $3 \%$ were excluded.

\section{Data analysis}

The details of data analysis procedure have been presented previously. ${ }^{5}$ Briefly, regions of interest (ROIs) were drawn over the entire kidney, cortex, and background on each side. Background corrected time-activity curves were constructed for each kidney using both the renal and cortical ROIs.

The time-activity curves were analyzed quantitatively for clearance, percent of function in the left and right kidneys, and parenchymal transit time. Clearance, in terms of percent uptake, was determined using a camera based method without blood or urine sampling., $5-9$ The background corrected counts for each kidney in the 1-2 minute image were corrected for attenuation using the method of Schlegel to give the percent dose cleared by each kidney at 1-2 minutes following injection. ${ }^{9}$ The result is inherently normalized for total blood volume and, therefore, body surface area. 
The percent of total renal function or clearance in each kidney was determined from the percent dose values. The parenchymal transit times were calculated from the peak activity times and from the peak to one half of the peak times or half times in the renal and cortical time-activity curves.

\section{Statistical analysis}

The Student's $t$-test was used to compare results between groups and the linear correlation coefficient was used when data were analyzed across all patients without regard to group. Interpatient variability for each parameter was determined from the absolute percent change between the first study in each patient and the expected value. The expected value was determined by averaging the first and second study for all patients in the same time group and then calculating the average for the group. Intrapatient variability for each parameter was determined from the ratio of the first and second studies calculated in absolute percentage terms.

\section{Results}

\section{Patient data}

Two patients were excluded and replaced because of extravasation of the dose in one and an unexpected horseshoe kidney in the other. The mean, standard deviation, and range of age by patient group, duration of injury by level of injury and patient group, serum creatinine, time between paired studies, and percent dose infiltration in the first and second studies are given in Table 1. All patients were male and between 20 and 40 years of age; there was no correlation between age and time from SCI. Ten patients were tetraplegic and 10 paraplegic with at least two tetraplegic and two paraplegic patients in each group. All but two patients had indwelling catheters at the time they were studied. The cortical ROI data in one of the two studies were not available in five patients for technical reasons; these missing data occurred at least once in each patient group.

\section{Renal clearance}

There was no correlation between renal clearance and patient age (Figure 1). In addition, there was no significant difference in renal clearance between the left and right kidneys in any group (Table 2). Renal clearance in the less than 2 month group was not significantly different from normal subjects for either paraplegic or tetraplegic patients. However, clearance in the tetraplegic patients was increased by $28.5 \%$ and $50.6 \%$ in the 2 to 12 month and 1 to 2 year groups, respectively, and was decreased by $25.9 \%$ in the greater than 2 year group compared to normal subjects (all $P<0.02$ ) (Figure 2). Clearance in those who were paraplegic showed a similar, but less marked, trend $(P=\mathrm{NS})$. The differences between those with tetraplegia and those with paraplegia in the 1 to 2 year and greater than 2 year groups were significant $(P<0.04)$. However, the duration of injury is on average much longer in the tetraplegic patients in the greater than 2 year group.

Table 1 Patient and study data

\begin{tabular}{|c|c|c|c|}
\hline Parameter/group & $\mathrm{n}$ & Mean \pm s.d. & Range \\
\hline \multicolumn{4}{|l|}{ Patient age (year) } \\
\hline Less than 2 months from SCI* & 5 & $30.8 \pm 6.1$ & $24-39$ \\
\hline 2 to 12 months from SCI & 5 & $28.8 \pm 7.1$ & $21-36$ \\
\hline 1 to 2 years from SCI & 5 & $29.2 \pm 6.6$ & $23-37$ \\
\hline Greater than 2 years & 5 & $32.4 \pm 3.5$ & $27-36$ \\
\hline \multicolumn{4}{|l|}{ Time from SCI } \\
\hline \multicolumn{4}{|l|}{ Less than 2 months from SCI (month) } \\
\hline Tetraplegics & 3 & $1.3 \pm 0.3$ & $1.1-1.7$ \\
\hline Paraplegics & 2 & $1.5 \pm 0.3$ & $1.3-1.7$ \\
\hline \multicolumn{4}{|l|}{2 to 12 months from SCI (month) } \\
\hline Tetraplegics & 2 & $2.6 \pm 0.5$ & $2.2-2.9$ \\
\hline Paraplegics & 3 & $2.5 \pm 0.5$ & $2.1-3.1$ \\
\hline \multicolumn{4}{|l|}{1 to 2 years from SCI (year) } \\
\hline Tetraplegics & 2 & $1.4 \pm 0.2$ & $1.2-1.6$ \\
\hline Paraplegics & 3 & $1.7 \pm 0.2$ & $1.5-1.9$ \\
\hline \multicolumn{4}{|l|}{ Greater than 2 years (year) } \\
\hline Tetraplegics & 3 & $8.8 \pm 5.5$ & $2.8-13.6$ \\
\hline Paraplegics & 2 & $2.6 \pm 0.7$ & $2.1-3.1$ \\
\hline Serum creatinine $(\mathrm{mg} / \mathrm{dL})$ & 20 & $0.8 \pm 0.2$ & $0.5-1.0$ \\
\hline Time between studies (day) & 20 & $7.3 \pm 10.4$ & $2-44$ \\
\hline Dose infiltration - first study (\%) & 20 & $0.7 \pm 0.3$ & $0.8-1.2$ \\
\hline Dose infiltration - second study $(\%)$ & 20 & $1.0 \pm 0.4$ & $0.3-2.0$ \\
\hline
\end{tabular}

* SCI: spinal cord injury 


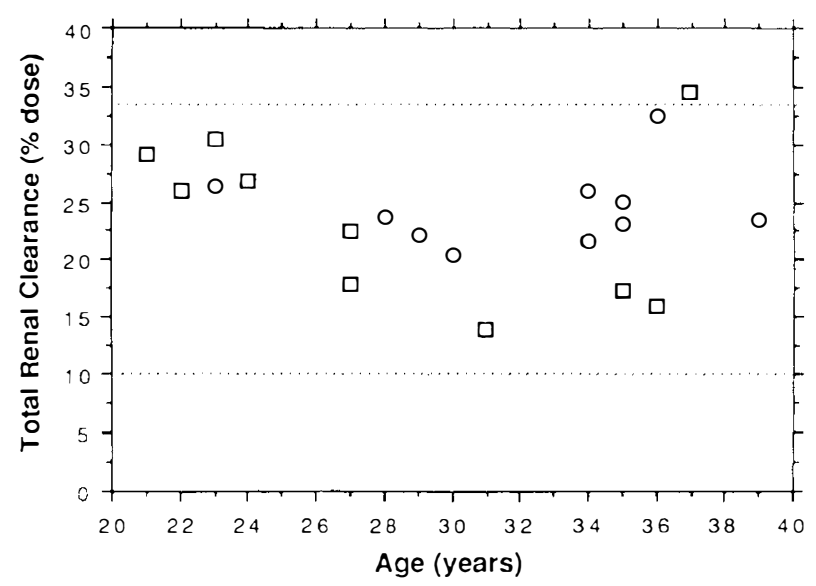

Figure 1 Total renal clearance is plotted against patient age; open squares represent tetraplegics and open circles represent paraplegics. The normal range (mean \pm 2 s.d.) is shown as broken lines. There is no significant correlation between total renal clearance and age for either tetraplegics or paraplegics

\section{Other parameters}

For the other physiological parameters, there were no significant differences between left and right kidneys, between those who were paraplegic and those who were tetraplegic among the four groups (Table 3). However, the left kidney peak time, and the left and right cortical peak times for all groups combined were significantly longer than normal subjects by the unidirectional $t$-test $(P<0.05)$. In addition, as in normal subjects the results of peak times with cortical ROIs were significantly less than with renal ROIs for all groups combined $(P<0.05)$.

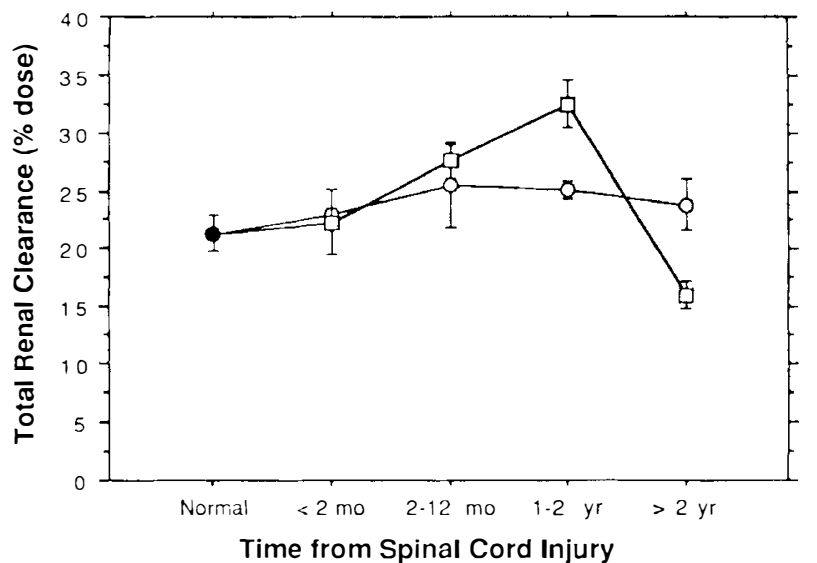

Figure 2 Total renal clearance as percent dose at 1-2 minutes after injection (mean \pm s.e.m.) is shown for normal subjects age $20-40$ years (solid circles, previously published in reference 5), and for tetraplegics (open squares) and paraplegics (open circles) for each of four groups of patients with varying durations of spinal cord injury. The average clearance for the two studies in each subject or patient was used in calculating the results. For tetraplegics compared to normal subjects the increase in the 2-12 month and 1-2 year groups and the decrease in the greater than 2 year group are all significant $(P<0.02)$. The similar, but much less marked, trend in paraplegics is not statistically significant

\section{Reproducibility}

The percent standard deviation for renal clearance in serial studies in the same patient (test-retest) was less than in single studies (between patients), but the decrease was significant for only the right kidney $(P<0.03)$ and the decrease was not as great as in normal subjects. Also, there was a trend toward less reproducibility in paraplegic patients compared to

Table 2 Renal clearance ( $\%$ dose)*

\begin{tabular}{|c|c|c|c|c|}
\hline Group & $\mathrm{n}$ & Left & Right & Total \\
\hline Normal subjects $\ddagger$ & 12 & $10.8 \pm 2.9$ & $10.7 \pm 2.7$ & $21.5 \pm 5.6 \dagger$ \\
\hline \multicolumn{5}{|l|}{ Less than 2 months } \\
\hline Tetraplegics & 3 & $11.4 \pm 2.4$ & $10.6 \pm 2.1$ & $22.0 \pm 4.1$ \\
\hline Paraplegics & 2 & $10.2 \pm 3.3$ & $9.4 \pm 1.8$ & $19.6 \pm 5.1$ \\
\hline \multicolumn{5}{|l|}{2 to 12 months } \\
\hline Tetraplegics & 2 & $15.1 \pm 2.2$ & $14.5 \pm 1.8$ & $29.5 \pm 0.4 \dagger$ \\
\hline Paraplegics & 3 & $12.5 \pm 4.9$ & $14.8 \pm 6.3$ & $27.2 \pm 11.1$ \\
\hline \multicolumn{5}{|l|}{1 to 2 years } \\
\hline Tetraplegics & 2 & $15.6 \pm 0.6$ & $18.4 \pm 5.2$ & $34.0 \pm 5.7 \dagger$ \\
\hline Paraplegics & 3 & $12.6 \pm 1.5$ & $13.9 \pm 3.8$ & $26.5 \pm 5.3 \dagger$ \\
\hline \multicolumn{5}{|l|}{ Greater than 2 years } \\
\hline Tetraplegics & 3 & $7.9 \pm 2.2$ & $6.8 \pm 1.5$ & $14.7 \pm 3.6 \dagger$ \\
\hline Paraplegics & 2 & $11.7 \pm 1.6$ & $10.9 \pm 1.6$ & $22.6 \pm 3.1 \dagger$ \\
\hline
\end{tabular}

* Data are presented as percent of injected dose in the kidney(s) at 1-2 minutes after injection (mean \pm s.d.). All data are from the first study in each patient. $¥$ These data have been previously published in reference 5 . $†$ The differences between total clearance for normal subjects and for the 2-12 month, 1-2 year, and greater than 2 year groups for tetraplegics are all significant $(P<0.02)$. The differences between total clearance for tetraplegics and paraplegics at $1-2$ years and greater than 2 year groups are both significant $(P<0.04)$ 
tetraplegic patients (Figure 3). The correlation coefficient and regression equation for normals were 0.91 and $\mathrm{Y}=1.05+0.96 \mathrm{X}$; for tetraplegic patients they were 0.90 and $\mathrm{Y}=8.03+0.65 \mathrm{X}$; and for paraplegic patients they were -0.22 and $\mathrm{Y}=26.4-0.08 \mathrm{X}$.

The percent standard deviation in serial studies for percent function in each kidney was significantly less than the percent standard deviation in single studies $(P<0.02)$ although again the difference was not as great as in normal subjects. There was no significant difference in intra- versus interpatient reproducibility in any of the other parameters.

\section{Discussion}

This study delineates the normal range and reproducibility of several important physiological parameters of renal function in SCI patients who have no evidence of renal disease. Of the various parameters of renal function, clearance as measured in Tc-99m-MAG3 studies was the only one that varied with time from the date of injury. At 1 to 2 years following SCI tetraplegic patients had a $50 \%$ increase in renal clearance which then decreased below normal. The paraplegic patients showed a similar, but much less marked, trend.

Previous studies of renal clearance in SCI patients without evidence of renal disease have reported a variety of results. Using inulin and para-aminohippurate clearance measurements, Price et al. in 1966 reported normal results in SCI patients that did not decrease with duration of injury through 15 years. ${ }^{11}$ Kuhlemeier et al., using I-131-orthoiodohippurate, concluded that there was no significant change in renal clearance with duration of SCI for either tetraplegic or paraplegic patients although qualitatively their data did show a small rise at one year followed by a fall below inital values which is similar to the present data. ${ }^{12,13}$ Temkin et al. using I-131-orthoiodohippurate and Price et al. in 1975 using inulin and para-aminohippurate reported initially normal clearance that decreased in a normal

Table 3 Results for parameters other than clearance*

\begin{tabular}{lccc}
\hline Parameter & $\mathrm{n}$ & Left & Right \\
$\begin{array}{l}\text { Percent of total } \\
\quad \text { clearance (\%) }\end{array}$ & 20 & $49.9 \pm 3.9$ & $49.6 \pm 3.5$ \\
$\begin{array}{l}\text { Peak time } \\
\quad \text { Renal ROI (min) } \dagger\end{array}$ & 20 & $4.1 \pm 1.4 \ddagger$ & $4.2 \pm 1.9$ \\
$\quad$ Cortical ROI (min) $\dagger$ & 15 & $3.2 \pm 1.1 \ddagger$ & $3.2 \pm 0.9 \ddagger$ \\
Half time & & & \\
$\quad$ Renal ROI (min) & 20 & $4.9 \pm 1.6$ & $5.4 \pm 1.7$ \\
$\quad$ Cortical ROI (min) & 15 & $4.7 \pm 1.2$ & $5.0 \pm 2.1$ \\
\hline
\end{tabular}

* All data are from the first study in each patient and are given as mean \pm s.d. $\dagger$ Differences between these two parameters are statistically significant $(P<0.05)$. $\ddagger$ Differences between these parameters and corresponding parameters in normal subjects are statistically significant $(P<0.05)$ fashion with age; no relation to duration of SCI for either tetraplegic or paraplegic patients was found. ${ }^{14,15}$ The reason that we detected a significant change in renal
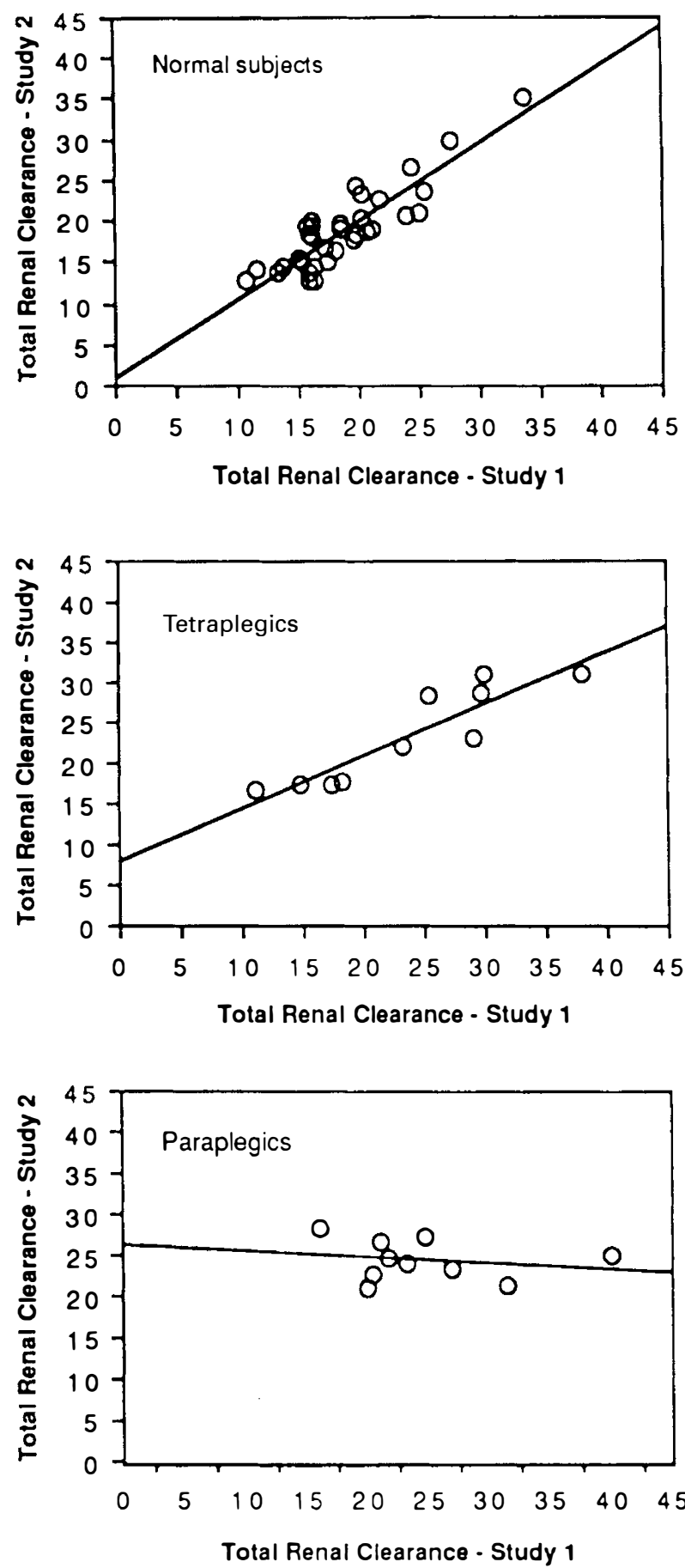

Figure 3 The scatterplots for total renal clearance measurements in the first and second Tc-99m-MAG3 renal studies in normal subjects, tetraplegics, and paraplegics are shown. The reproducibility in normal subjects is high with a y-intercept of almost zero, and a slope of nearly one. On the other hand the reproducibility in tetraplegics and especially paraplegics is much lower 
Table 4 Interpatient $v s$ intrapatient variability $(\%)^{*}$

\begin{tabular}{|c|c|c|c|c|}
\hline Parameter & $\mathrm{n}$ & Left & Right & Total \\
\hline \multicolumn{5}{|l|}{ Single studies } \\
\hline Clearance & 20 & $20 \pm 13$ & $28 \pm 19+$ & $20 \pm 13$ \\
\hline Percent of total clearance $\dagger$ & 20 & $5 \pm 4$ & $5 \pm 3$ & - \\
\hline Peak time (renal ROI) & 20 & $25 \pm 25$ & $29 \pm 14$ & - \\
\hline Half time (renal ROI) & 20 & $25 \pm 17$ & $26 \pm 14$ & - \\
\hline Peak time (cortical ROI) & 15 & $28 \pm 17$ & $38 \pm 58$ & - \\
\hline Half time (cortical ROI) & 15 & $20 \pm 14$ & $29 \pm 44$ & - \\
\hline \multicolumn{5}{|l|}{ Serial studies } \\
\hline Clearance & 20 & $16 \pm 18$ & $17 \pm 17 \dagger$ & $16 \pm 17$ \\
\hline Percent of total clearance $\dagger$ & 20 & $3 \pm 3$ & $3 \pm 3$ & - \\
\hline Peak time (renal ROI) & 20 & $37 \pm 44$ & $44 \pm 30$ & - \\
\hline Half time (renal ROI) & 20 & $24 \pm 20$ & $33 \pm 21$ & - \\
\hline Peak time (cortical ROI) & 15 & $40 \pm 41$ & $39 \pm 25$ & - \\
\hline Half time (cortical ROI) & 15 & $30 \pm 32$ & $38 \pm 55$ & - \\
\hline
\end{tabular}

* The data indicate the percent change (mean \pm s.d.) between individual first studies and the average of first studies in the same group for interpatient variability, and from the first to the second study for intrapatient variability. $\dagger$ Differences between intrapatient and interpatient variability for clearance in the right kidney (but not the left kidney nor total clearance) and for percent of total clearance on left and right were statistically significant $(P<0.03)$

clearance with duration of injury in tetraplegic patients while others have not is uncertain, but may be related to better temporal resolution early after injury in our study. We have previously shown that our method of measuring renal clearance with Tc-99m-MAG3 shows the expected decline in clearance with age in normal subjects. 5

The initial rise in renal clearance that we have measured theoretically may be secondary to either an increase in renal clearance of Tc-99m-MAG3 or to a decrease in the effective volume of distribution of Tc99m-MAG3. Renal clearance in turn is a function of renal blood flow times the extraction efficiency of tubular cells for Tc-99m-MAG3. The effective volume of distribution is determined by the degree to which Tc-99m-MAG3 binds to blood cells and plasma proteins, and the degree to which it diffuses into the interstitial space. Kolb et al. review evidence that the volume of distribution of various drugs may be increased in SCI patients at the same time that their renal clearance of certain drugs is also increased. ${ }^{16}$ If renal clearance of a drug is truly increased in the face of an enlarged volume of distribution, then renal clearance must be increased to an even greater degree than the increase in the volume of distribution.

Possible reasons for a temporary change in renal clearance and/or effective volume of distribution of Tc-99m-MAG3 would include direct effects of SCI on sympathetic regulation of renal physiology and/or the size of the extracellular space. The kidney is known to have a rich sympathetic innervation. ${ }^{17}$ Another possible explanation is muscle catabolism following SCI. ${ }^{18}$ It is known that increased plasma levels of certain amino acids, particularly glycine and alanine, secondary to ingesting meat increases renal clearance by as much as $50 \%{ }^{19-23}$ Both tetraplegic and paraplegic patients experience muscle wasting of the lower extremities following SCI. However, in those with tetraplegia there is also wasting of the upper extremities while in paraplegic patients the upper extremities hypertrophy with compensatory increased use. These differences between tetraplegic and paraplegic patients could explain the differences in renal function with time from injury in these two types of patients. Tetraplegic individuals presumably release a large amount of amino acids early after injury followed by below normal release later because of reduced muscle mass. On the other hand, paraplegic patients presumably release a smaller amount of amino acids initially and maintain a more normal overall muscle mass long term.

Quantification of renal clearance with radiopharmaceuticals may be performed in many different ways. In the present study a camera based method, without blood or urine sampling, was chosen because it does not require bench top chemistry skills and can be more easily instituted in most hospitals. ${ }^{24}$ Some reduction in accuracy may occur as a result of the increased convenience. $^{25} 28$ In addition, we elected to express renal clearance in terms of the percent dose cleared by the kidneys at $1-2$ minutes after injection rather than the traditional ERPF in order to maximize simplicity and to minimize overall error. ${ }^{5,29}$

There were no differences in any of the parameters between the left and right kidneys. There were also no differences among the four patients groups or between tetraplegic and paraplegic patients in percent function on a side, peak time, or half time following the peak. However, the peak times for both kidneys using cortical ROIs and for the left kidney using a renal ROI were significantly longer than in normal subjects. This finding is most likely secondary to the fact that SCI patients frequently have mildly dilated renal collecting systems even without evidence of renal 
disease. ${ }^{12}$ In addition, the peak times were shorter with cortical ROIs than with kidney ROIs, presumably because the latter include the proximal collecting systems. The same finding was found in normal subjects and suggests that cortical ROIs should be used in preference to whole kidney ROIs in order to more accurately measure parenchymal transit times. ${ }^{5}$

Serial renal studies are performed routinely in SCI patients. In this situation it is important to know the normal limits for change in serial measurements in the same patient rather than single measurements because the range is likely to be less, increasing the sensitivity of the test. Our results indicate that the percent standard deviation for serial studies of renal clearance and percent of function in each kidney were in most cases less than in single studies in different patients. However, the decrease in variation was not as great as in normal subjects. ${ }^{5}$ In addition, for unknown reasons paraplegic patients showed much less reproducibility than did tetraplegic patients. These findings suggest that SCI patients are more difficult to study reproducibly than normal subjects.

Unfortunately, the relatively large normal range, poor test re-test reproducibility (particularly in paraplegics), variation in tetraplegic individuals with time from injury, and the effect of diet make quantitation of total renal clearance a difficult parameter to use in the detection of renal disease in SCI patients. However, in most circumstances asymmetry in clearance (percent clearance on a side), presence of cortical scars in high contrast Tc-99mMAG3 images, and abnormal collecting systems are probably more sensitive to early disease than changes in total renal clearance. This is particularly true since the effects of infection and obstruction are unlikely to be symmetrical and uniform.

We conclude that renal clearance measured with Tc99m-MAG3 in tetraplegic patients without evidence of renal disease increases by approximately $50 \%$ during the first 2 years following injury and decreases to below normal thereafter, that peak times with cortical ROIs are longer for SCI patients than for normal subjects, and there is somewhat more intrapatient variation in measurements of total renal clearance and percent renal clearance on a side in SCI patients than in normal subjects suggesting that it is harder to study SCI patients reproducibly. These findings should be helpful in performing and interpreting Tc-99m-MAG3 renal studies in SCI patients.

\section{Acknowledgements}

This study was supported in part by a grant from Mallinckrodt Medical, St. Louis, Missouri, USA.

\section{References}

1 Vera A, Merritt JL, Erickson BR. Renal function in spinal cord injury: A preliminary report. Arch Phys Med Rehabil 1986; 67: $257-259$.
2 Stover SO. Renal function tests on spinal cord injury patients. In Research Update, Spain Rehabilitation Center, Birmington, AL, USA, 1990

3 Lloyd AL, Dubovsky CVD, Bueschen AJ et al. Comprehensive renal scintillation procedures in spinal cord injury: Comparison with excretory urography. J Urol 1981; 126: 10-13.

4 Eshima D, Taylor A. Technetium-99m (99mTc) mercaptoacetyltriglycine: Update on the new $99 \mathrm{mTc}$ renal tubular function agent. Sem Nucl Med 1992; 22: 61-73.

5 Klingensmith WC, Briggs DE, Smith WI. Technetium-99mMAG3 renal studies: Normal range and reproducibility of physiologic parameters as a function of age and sex. $J \mathrm{Nucl}$ Med 1994; 35: $1612-1617$.

6 Klingensmith WC, Tyler HN, Marsh WE, et al. Effect of hydration and dehydration on technetium-99m CO2 DADS renal studies in normal volunteers. J Nucl Med 1985; 26: 875879.

7 Gates GF. Glomerular filtration rate: Estimation from fractional renal accumulation of Tc-99m-DTPA (stannous). Am J Roentgenol 1982; 138: $565-570$.

8 Gates GF. Split renal function testing using Tc-99m DTPA: A rapid technique for determining differential glomerular filtration. Clin Nucl Med 1983; 8: 400-407.

9 Schlegel JU, Hamway SA. Individual renal plasma flow determination in 2 minutes. $J$ Urology 1976; 116: $282-285$.

10 Harbert JC, Curl FB, Jones GW. The effect of injection volume and technique on radionuclide cerebral angiograms. J Nucl Med 1973; 14: 205-207.

11 Price M, Tobin JA, Reiser M, et al. Renal function in patients with spinal cord injuries. Arch Phys Med Rehabil 1966; 47: $406-$ 411.

12 Kuhlemeier KV, McEachran AB, Lloyd AL, et al. Renal function after acute and chronic spinal cord injury. J Urology 1984; 131: $439-445$.

13 Kuhlemeier KV, Lloyd AL, Stover SO. Long-term followup of renal function after spinal cord injury. J Urology 1985; 134: $510-$ 513.

14 Tempkin A, Sullivan G, Paldi J, et al. Radioisotope renography in spinal cord injury. J Urology 1985; 133: $228-230$.

15 Price M, Kottke FJ, Olson ME. Renal function in patients with spinal cord injury: The eighth year of a ten-year continuing study. Arch Phys Med Rehabil 1975; 56: 76-79.

$16 \mathrm{Kolb} \mathrm{KW}$, Ha CY. Pharmacokinetic and pharmacodynamic alterations in drug therapy caused by spinal cord injury. Phys Med Rehabil 1987; 1: 375-388.

17 Rabkin R, Dahl DC. Hormones and the kidney. In Diseases of the Kidney, RW Schrier \& CW Gottschalk, eds, Little, Brown and Co, Boston, 1993, pp 283-303.

18 Nuhlicek CN, Spurr GB, Barboriak JJ, et al. Body composition of patients with spinal cord injury. Eur J Clin Nutr 1988; 42: 765 773.

19 Nakamura $\mathrm{H}$, Ebe N, Ito S, et al. Renal effects of different types of protein in healthy volunteer subjects and diabetic patients. Diabetes Care 1993; 16: 1071 -- 1075.

20 Hostetter TH. Human renal response to a meat meal. Am J Physiol 1986; 250: F613-618.

21 Bosch JP, Saccaggi A, Lauer A, Ronco C, et al. Renal function reserve in humans. Am J Med 1983; 75: $943-950$.

22 Nair KS, Pabico RC, Truglia JA, et al. Mechanism of glomerular hyperfiltration after a protein meal in humans. Diabetes Care 1994; 17: $711-715$.

23 Wiseman MJ, Hunt R, Goodwin A, et al. Dietary composition and renal function in healthy subjects. Nephron 1987; 46: 37-42.

24 Russell CD, Dubovsky CVD. Measurement of renal function with radionuclides. $J$ Nucl Med 1989; 30: 2053-2057.

25 Russell CD, Thorstad BL, Yester MV, et al. Quantitation of renal function with technetium-99m MAG3. J Nucl Med 1988; 29: $1931-1933$.

26 Prenen JAC, de Klerk JMH, van het Schip $\mathrm{AD}$, et al. Technetium-99m-MAG3 versus iodine-123-OIH: Renal clearance and distribution volume as measured by a constant infusion technique. J Nucl Med 1991; 32: 2057-2060. 
27 Fine EJ, Axelrod M, Gorkin J, et al. Measurement of effective renal plasma flow: A comparison of methods. J Nucl Med 1987; 28: $1393-1400$.

28 Russell CD, Dubovsky CVD. Quantitation of renal function using MAG3. J Nucl Med 1991; 32: 2061-2063.
29 Berriolo A, Touzery C, Riedinger JM, et al. Estimation of Tc99m-MAG3 plasma clearance from the global renal uptake measured with a gamma camera. Eur J Nucl Med 1991; 32: 619. 